\title{
Phase-locked eduction of vortex shedding in flow past an inclined flat plate
}

\author{
K. M. Lam \\ Department of Civil and Structural Engineering, The University of Hong Kong, Pokfulam Road, \\ Hong Kong
}

(Received 13 June 1995; accepted 18 January 1996)

\begin{abstract}
Flow past an inclined flat plate at an angle of attack of $30^{\circ}$ and a Reynolds number of 30000 is investigated experimentally. The velocity field in the wake is measured with a laser doppler anemometer (LDA) in the region from one plate breadth downstream to three and a half-plate breadths downstream. Controlled forcing is applied to the wake by vibrating the plate in the across-wind direction at a frequency in the middle of the lock-in range. The forcing serves to enhance the regularity and two-dimensionality of vortex shedding from the plate. It also facilitates phase-locked averaging of the LDA data. The LDA bursts are sorted according to their arrival instants relative to a particular phase of the vortex shedding cycles. The phase-averaged velocity results reveal large-scale vortical structures in the wake. Dynamical properties of these structures such as coherent vorticity and Reynolds stress production are discussed. The wake is found to be strongly asymmetric. The flow dynamics in the wake are dominated by a train of counterclockwise vortices shed from the trailing edge of the plate. The development, shedding and subsequent convection of these vortices are studied by following the consecutive phases of the shedding cycle.

(C) 1996 American Institute of Physics. [S1070-6631(96)00105-3]
\end{abstract}

\section{INTRODUCTION}

Vortex shedding is an important feature of bluff body aerodynamics. The shedding of vortices and their dynamics are believed to play a governing role in many features of the flow such as fluid mixing, heat transfer, and noise generation. Many believe that vortex shedding occurs at all practical Reynolds numbers, ranging from the classical vonKarman vortex street in laminar flow over a circular cylinder to highly turbulent wind flow past a mountain. In turbulent flows over bluff bodies, perfect regularity of vortex shedding is impaired and the large-scale vortices or coherent structures are embedded in the incoherent turbulence. The phaselocked eduction technique has proven to be a useful tool in revealing the coherent vortical structures in turbulent flows. ${ }^{1}$ To use the eduction technique successfully in the near wake behind a bluff body, one has to be able to make reliable measurements of the flow velocities in this highly turbulent and perhaps recirculating flow region. The development of techniques, such as the flying hot wire and laser doppler anemometer (LDA), has made this possible. With the flying hot wire and the phase-locked eduction technique, Cantwell and Coles ${ }^{2}$ made a comprehensive study of the vortex dynamics in flow over a circular cylinder. Phase-locked LDA measurements of flow past a vertical flat plate was reported by Leder. ${ }^{3}$

In two-dimensional bluff body flows, the circular cylinder and the flat plate normal to the flow receive most attentions. It is well established that there are two shear layers of equal strength which lead to the shedding of a symmetric vortex street. For flow past an inclined flat plate, the flow geometry and the mean velocity field in the very near wake of recirculating region are clearly asymmetric. It is interesting to investigate whether the subsequent vortex shedding and vortex development also bear asymmetric characteristics. The earliest work on this topic was that of Fage and Johansen, ${ }^{4}$ who investigated vortex shedding from a flat plate at flow incidence from normal to almost parallel. Based on velocity measurements made in the wake, they showed that the vortex shedding frequency scaled with the windward width of the plate, $D^{\prime}=D \sin \alpha$. This constancy of Strouhal number $f D^{\prime} / U_{0}$ was observed from the angle of attack $\alpha=90^{\circ}$, that is normal incidence, to $\alpha=30^{\circ}$. At $\alpha$ below $30^{\circ}$, the Strouhal number became higher, an observation made again recently by Knisely. ${ }^{5}$ Based on mean velocity and turbulence intensity profiles made in the wake from $\alpha=90^{\circ}$ to $30^{\circ}$, Fage and Johansen ${ }^{4}$ showed that vorticity was shed from both edges of the flat plate at the same rate. Sarpkaya ${ }^{6}$ used the discrete vortex method to model the wake of inclined flat plates and showed the presence of large-scale vortices on both sides of the near wake. However, the confident results of these early studies were made at $\alpha$ above $30^{\circ}$ and the possibly different development and dynamics of the shed vortices from the two plate edges had not been the focus of study.

In a more recent work by Perry and Steiner, ${ }^{7}$ detail vortex shedding process from a flat plate inclined at $45^{\circ}$ was studied experimentally with phased-averaged velocity measurements using a flying hot wire. The mean velocity vector field immediately behind the inclined plate was clearly shown to be highly asymmetric. A careful examination of the time-dependent phase-averaged vector fields and streamline patterns over a shedding cycle suggests that vortices shed from the trailing edge and the leading edge seem to be of unequal strength. Unfortunately, other dynamical properties of the vortices such as phase-averaged vorticity and Reynolds stresses were not reported for the inclined plate in that study. Similar to flow past an inclined flat plate is the flow over an aerofoil. At small angles of attack, the aerofoil theory assumes only one train of vortices shed from the trailing edge, a postulation supported by flow visualizations. However, in that situation flow does not separate at the aerofoil nose. The flow at stall angles should be more relevant to the inclined plate situation. McAlister and $\mathrm{Carr}^{8}$ reported flow visualization pictures in a water tunnel of an aerofoil 
under dynamic stall at $\alpha$ between $10^{\circ}$ and $20^{\circ}$. These visualization pictures, also collected in the recent monograph by Naudascher and Rockwell, ${ }^{9}$ showed two trains of different strength vortices in a street behind the aerofoil. Another asymmetric wake geometry of possible relevance is the flow over an inclined disc reported by Calvert. ${ }^{10}$ Regular shedding of only one train of coherent vortices from the trailing edge was observed for all oblique flow incidence angles on the disk starting from slight departure from normal incidence.

It appears from these more recent investigations that quantitative data are not available on the dynamical features of the vortices shed from an inclined plate. There still remains the uncertainty whether the two shear layers at the two edges of the plate are of unequal strengths and whether one of the two vortex trains is dominant over the other. The uncertainty is particularly relevant to small angles of attack below $30^{\circ}$. The experimental investigation to be reported in this paper is aimed at obtaining this information. LDA measurements are made in the wake of an inclined flat plate at $\alpha=30^{\circ}$, and the development of vortices is studied with the phase-locked eduction technique.

\section{EXPERIMENTAL TECHNIQUES}

The experiments were carried out in a blow-down wind tunnel whose test section was $1 \mathrm{~m}$ long and hexagonal in cross section of width $0.45 \mathrm{~m}$. A flat plate machined with sharp edges was used and it had a width $D=40 \mathrm{~mm}$ and a length $L=440 \mathrm{~mm}(L / D=11)$. The flat plate was mounted horizontally, at $30^{\circ}$ to the oncoming flow, in the test section at a distance of $0.35 \mathrm{~m}$ from the end of the contraction section of the wind tunnel. Most experiments were performed at a free-stream wind velocity $U_{0}=10.9 \mathrm{~m} / \mathrm{s}$, measured at about $0.25 \mathrm{~m}$ upstream of the plate. The turbulence intensity was below $1 \%$. The Reynolds number based on $U_{0}$ and the plate width $D$ was about $3 \times 10^{4}$. The blockage is about 0.05 .

Flow velocities were measured with a two-color fiberoptic LDA using a $3 \mathrm{~W}$ Argon-ion laser. The LDA operated in the backscatter mode and with frequency shift. The doppler bursts were analyzed by a correlation-technique LDA signal processor (DANTEC model 58N20 FVA). The processor had a single channel capability and was shared by the two-color doppler signals. LDA data of the two velocity components were thus not obtained with coincidence. Measurements were made in the wake of the plate in the region streamwise from $x / D=1.0$ to 3.5 and laterally from $y / D=$ -1.0 to 1.0. The fiber-optic probe was moved with a computer-controlled traverse table so that the measuring point covered the measurement region in the spanwise plane of symmetry on a grid of 0.1 by $0.1 D$ resolution. Measurements farther upstream could not be made due to the difficulty in seeding the flow there.

For phase-locked eduction, the LDA velocity data have to be stamped with phase information relative to a reference instant of the vortex shedding cycles. In situations where regular shedding occurs, the reference signal can be obtained with a trigger hot wire placed in a suitable location in the wake to detect and register vortex shedding. In this study, it was decided to subject the flow to a controlled forcing which served a dual purpose in organizing the periodicity of vortex

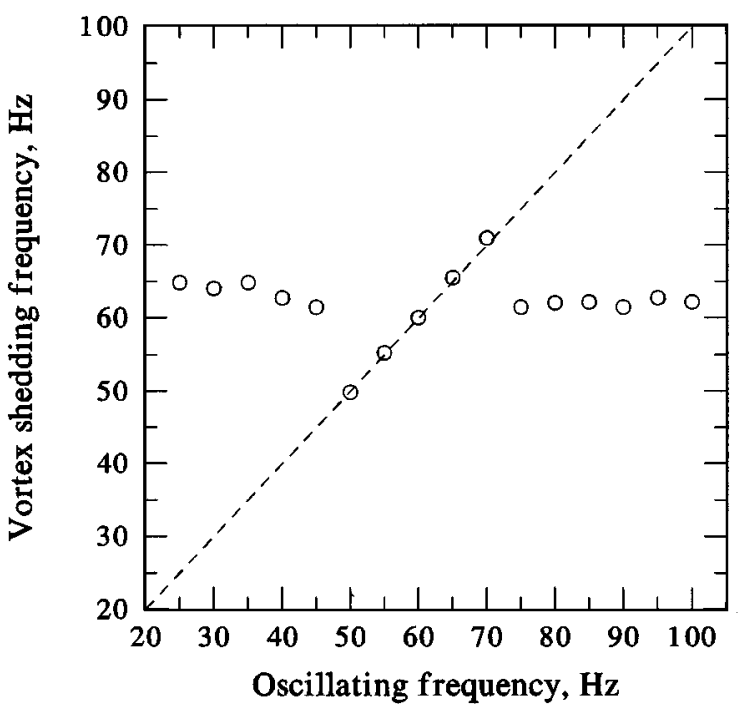

FIG. 1. Vortex shedding frequency versus plate oscillating frequency.

shedding as well as providing a convenient phase reference signal. Before the application of forcing, the vortex shedding frequency at $\alpha=30^{\circ}$ and $U_{0}=10.9 \mathrm{~m} / \mathrm{s}$ behind the plate was revealed by the hot-wire spectra in the wake to be about 64 Hz. This shedding frequency, corresponding to a Strouhal number $f D^{\prime} / U_{0}=0.12$, appears quite low as compared with the values found in earlier studies. For normal and inclined plates, Fage and Johansen ${ }^{4}$ measured values from 0.145 to 0.15 , which were repeated by Knisely ${ }^{5}$ at a much lower Reynolds number, whereas a Strouhal number of 0.14 was reported in Leder $^{3}$ for a vertical flat plate at nearly the same Reynolds number as the present study. The present low value of shedding frequency may be due to a poor twodimensionality of vortex shedding and the absence of end plates. Installation of end plates in the present hexagonal test section would largely reduce the aspect ratio of the plate. Perry and Steiner ${ }^{7}$ showed that a sacrifice of aspect ratio to allow for installation of end plates may not necessarily improve the situation. It will also be shown later that twodimensionality of the flow was enhanced significantly by the artificial forcing.

Controlled forcing was applied by vibrating the flat plate vertically with a minishaker (B \& K model 4810 ) at a small vibration amplitude at about $1 \mathrm{~mm}$, that is $0.025 \mathrm{D}$. The response of vortex shedding to the plate oscillating frequency was investigated by measuring the hot-wire spectra in the wake and obtaining the peak frequencies while varying the forcing frequency. The results are shown in Fig. 1, which bears close resemblance to the numerical results of $\mathrm{Li}$ et al. ${ }^{11}$ dealing with lock-in of an oscillating circular cylinder. The lock-in range of the inclined plate was found from 50 to 70 Hz. It appears that the natural shedding frequency of the stationary plate in the ideal two-dimensional situation will unlikely be much higher than $64 \mathrm{~Hz}$. The width of the lock-in range, when normalized by $64 \mathrm{~Hz}$, is from $0.8 \mathrm{f}$ to 1.1f. This range agrees well with the computed results of $\mathrm{Li}$ et al. ${ }^{11}$ Without solid knowledge of the natural vortex shedding frequency in the ideal two-dimensional case, it was thus 


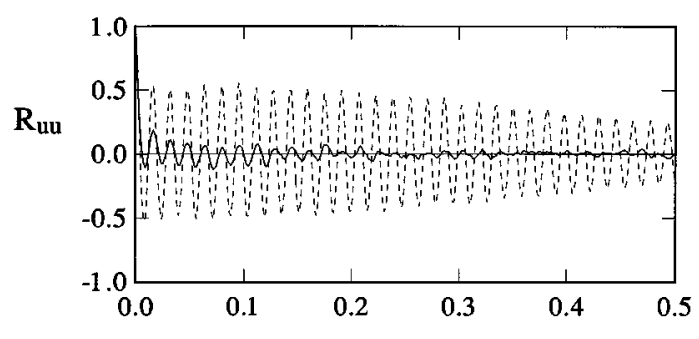

(a)

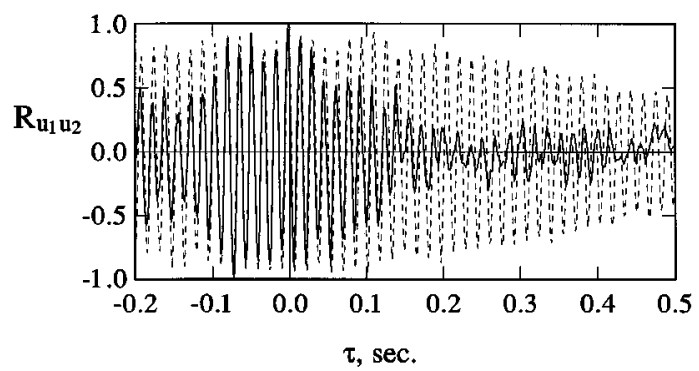

(b)

FIG. 2. Velocity correlations in the wake behind the trailing edge, $y / D \simeq$ -0.5 . (a) Auto correlation at midspan, $x / D=1.0$. (b) Cross correlation between midspan and $L / 3$ from midspan, $x / D=2.25$. — , stationary plate; -----, oscillating plate.

decided to apply the forcing at $64 \mathrm{~Hz}$. It is believed that with the plate oscillating at this middle frequency of the lock-in range, the dynamics of shed vortices are well representing of those of natural shedding. The effects of forcing are evident from Fig. 2 which shows some selected velocity correlations obtained with hot-wire measurements behind the trailing edge of the plate. The autocorrelations clearly show an organization of the periodicity of vortex shedding after forcing was applied. At the same time, two-dimensionality of the wake is greatly enhanced, as shown from the spanwise correlations.

Phase-locked eduction of the LDA data was performed as follows. A reference pulse was derived from each cycle of the sinusoidal signal which drove the minishaker. These reference pulses were fed into the LDA signal processor to provide the phase reset registers. Every validated LDA velocity data was thus labeled with the time lag from the previous phase reset. In this way, the LDA data at each measurement point, typical 1000 LDA bursts, were sorted into eight phases of the vortex shedding cycle. Ensemble averaging was then carried out on the velocity data at each phase to obtain the phase-averaged velocity. Following the decomposition scheme of Hussain, ${ }^{1}$ the flow velocity signal consists of a time-averaged component $\bar{U}$, a coherent component $u_{c}$ and an incoherent random component $u_{r}$. Ensemble averaging removes the random component and leaves the phaseaveraged velocity $\langle U\rangle$ which contains the mean and coherent components:

$$
U(t)=\bar{U}+u_{c}+u_{r}, \quad\langle U\rangle=\bar{U}+u_{c} .
$$

The technique is summarized schematically in Fig. 3.

\section{RESULTS AND DISCUSSION}

Time-averaged mean flow vectors in the wake are presented first in Fig. 4. The mean streamline pattern is obtained
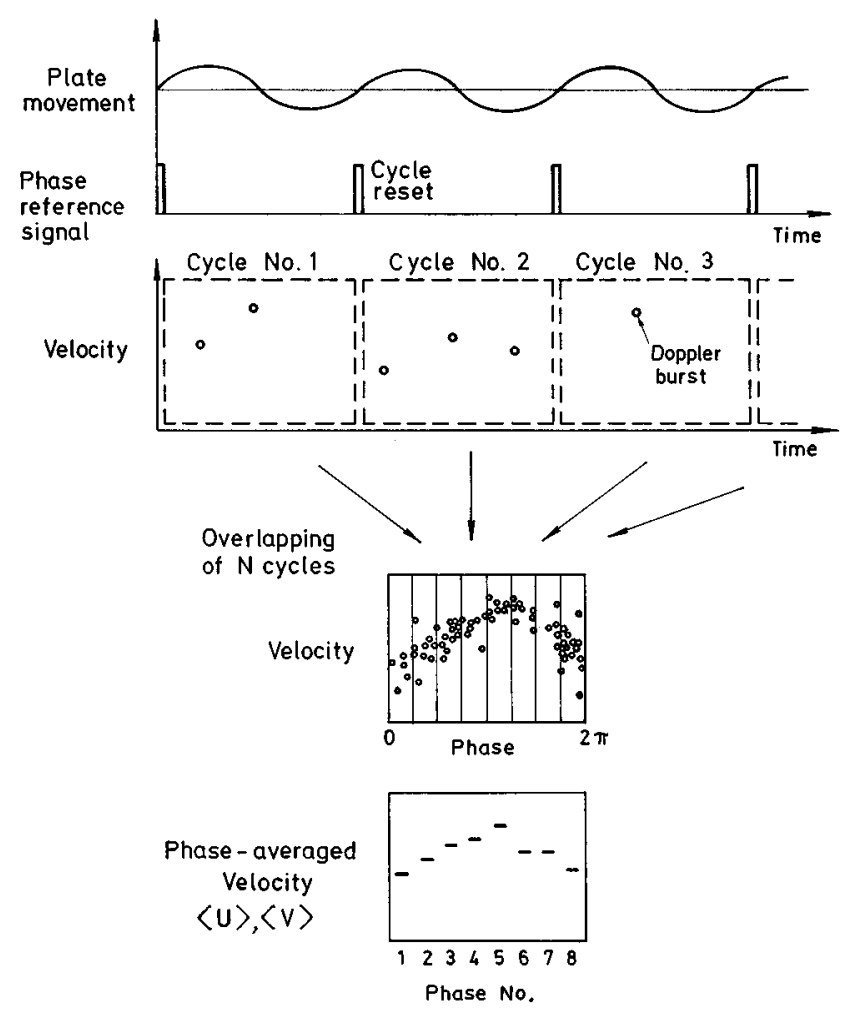

FIG. 3. Schematic diagram showing phase-locked eduction of LDA bursts.

from integration of the velocity vectors. Upstream of $x / D$ $<1.5$, flow reversal is found in the near wake behind the inclined plate, but regions of clearly circulating flow are not firmly observed with the lack of data upstream of $x / D<1.0$. However, there seems to be an asymmetry in flow reversal at the two sides of the plate in that a larger recirculating flow region is connected with the leading edge of the plate than the trailing edge. This is consistent with the observation made by Perry and Steiner ${ }^{7}$ for a $45^{\circ}$ inclined flat plate.

The dynamics of vortex shedding from the plate can be revealed by a number of flow quantities obtainable from the phase-locked LDA velocity data. First, Fig. 5 shows the phase-averaged velocity vectors $\langle U\rangle,\langle V\rangle$ at the eight phases of a shedding cycle. The starting phase is defined by the driving signal of the minishaker and is thus related to an unknown, but yet fixed, phase of vortex shedding. Phase-

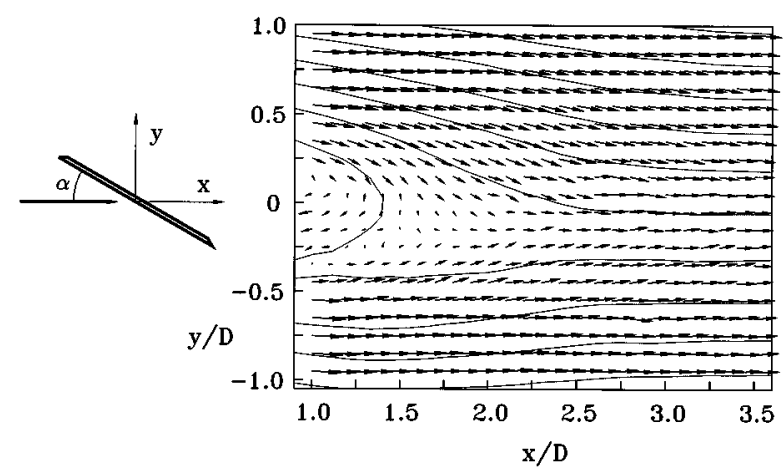

FIG. 4. Mean flow vectors and streamlines in the wake. 
Phase 1

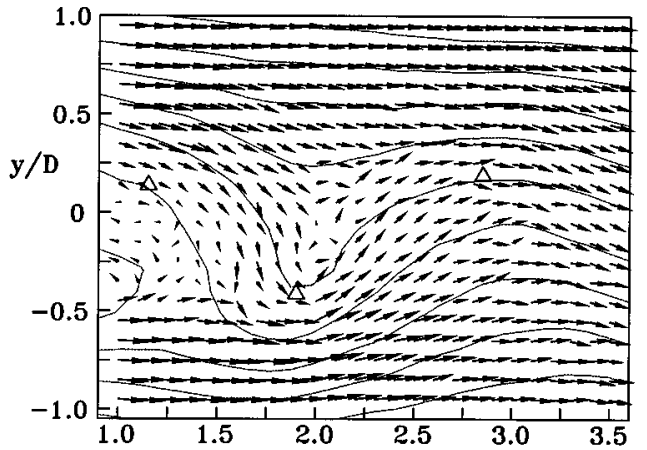

Phase 2

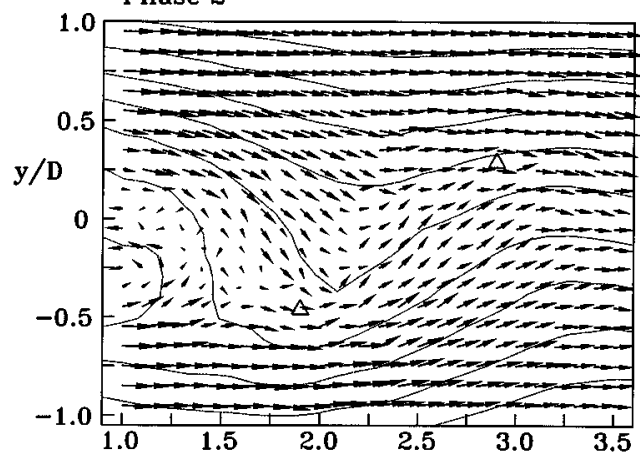

Phase 3

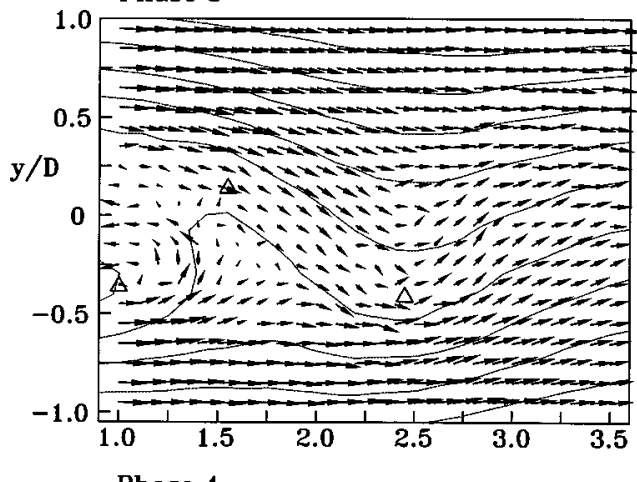

Phase 4

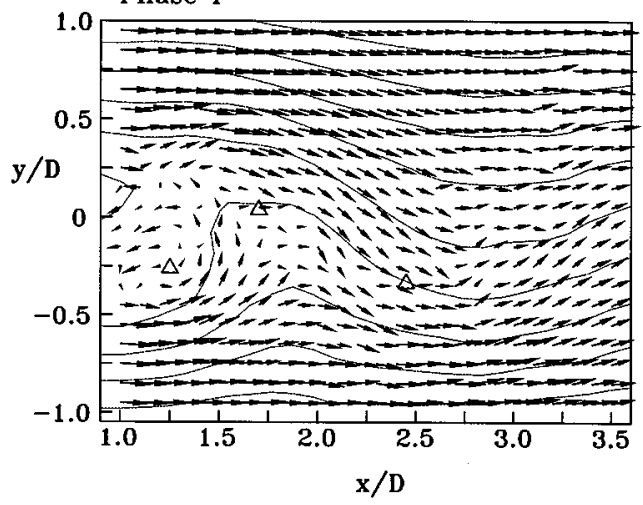

Phase 5

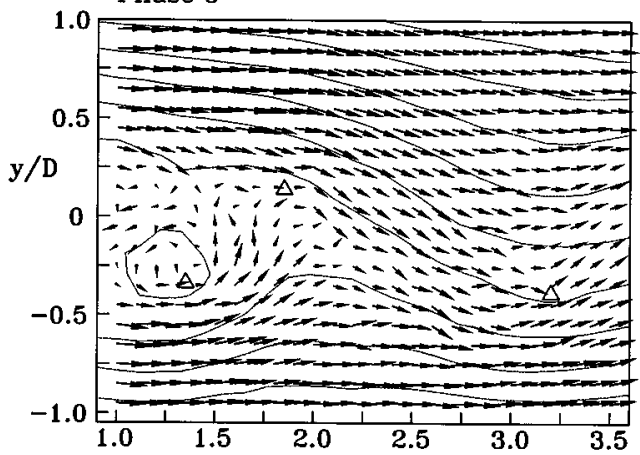

Phase 6

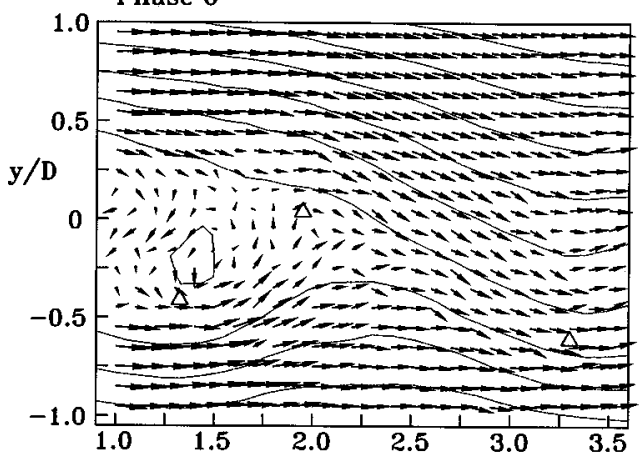

Phase 7

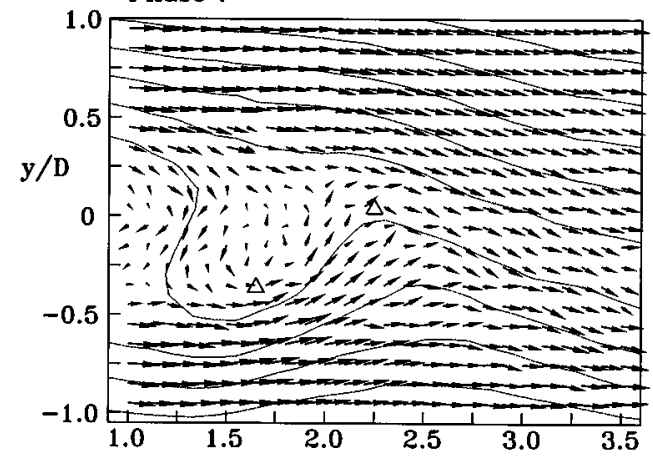

Phase 8

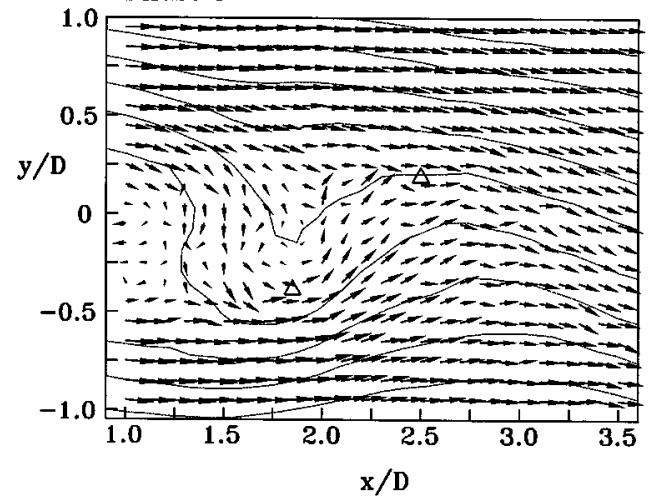

FIG. 5. Phase-averaged velocity vectors, $\langle U\rangle,\langle V\rangle$ and integrated streamline pattern. $\Delta$, location of peak positive or negative coherent vorticity.

averaged streamline patterns, obtained from integration of the velocity vectors, are superimposed on the vector plots in Fig. 5. Together, they describe the flow patterns induced by the convecting shed vortices in the wake as seen by a stationary observer. A better picture of the vortices can be obtained by making the observer moving with the vortices. The convection velocity of the vortices can be found by tracking the locations of vortices at successive times or phases. The vortices can be located by the fluid circulation they induced or, more quantitatively, by the vorticity values. The coherent vorticity in the wake field is computed from the phaseeduced velocities as 
Phase 1
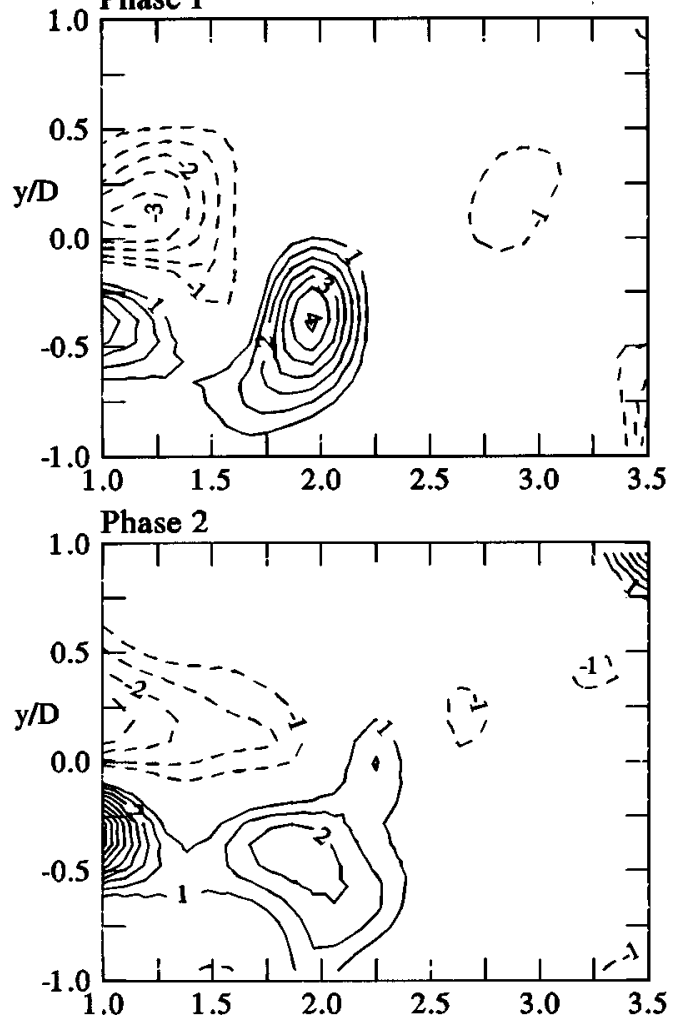

Phase 3
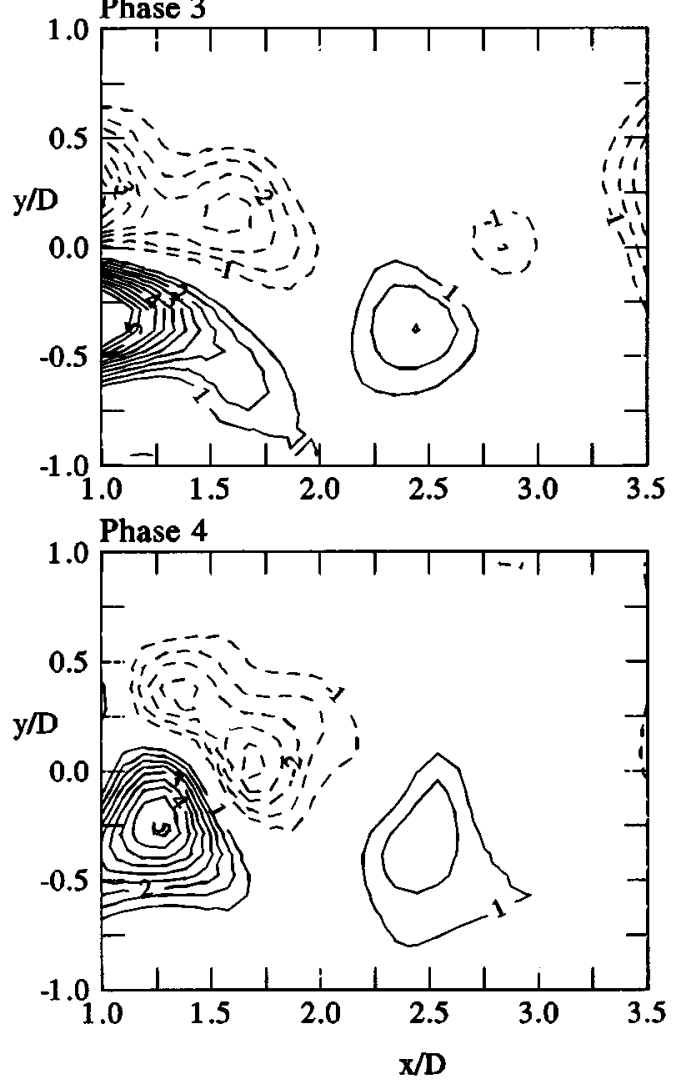

Phase 5
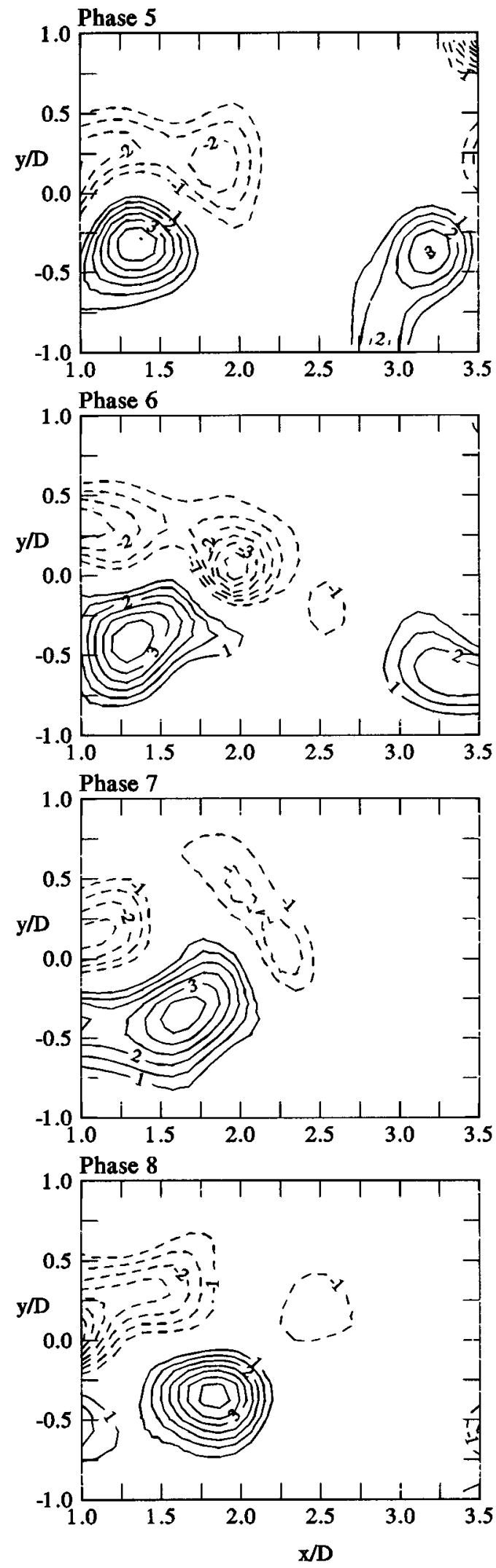

FIG. 6. Contours of coherent vorticity. Levels in $\Omega D / U_{0}$.

$\langle\Omega\rangle=\frac{\partial\langle V\rangle}{\partial y}-\frac{\partial\langle U\rangle}{\partial x}$.
Figure 6 shows the computed contours of coherent vorticity at the eight phases. The hills of peak positive vorticity on the 


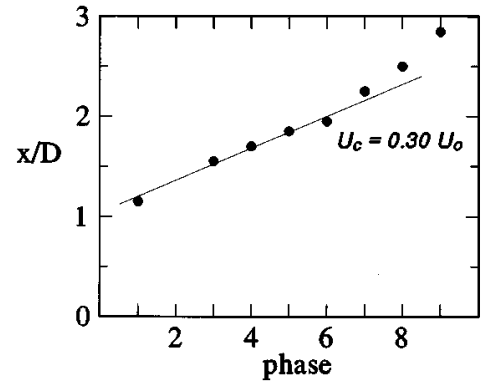

FIG. 7. Convection of clockwise vortices. Space-time locations from locations of peak coherent vorticity.

lower side of the wake are due to counterclockwise rotating vortices shed from the trailing edge of the plate. The valleys of negative vorticity are due to clockwise fluid rotations occurring on the upper side of the wake.

One can observe from Figs. 5 and 6 that the fluid circulations in the wake are dominated by the counterclockwise rotating vortices shed from the trailing edge of the plate. The convection of these vortices can easily be traced either by following the vorticity hill in Fig. 6 or by locating the center of fluid circulation from the vector plots in Fig. 5. The space-time convection of the counterclockwise vortex is shown in Fig. 7. When the vortex is within $x / D<2$, its convection velocity is about $U_{c}=0.30 U_{0}$. This low value of convection velocity may be because the vortex is in the process of detaching from the region of mean flow reversal. Farther downstream after it is completely shed, it is found to convect at a faster speed. Within $x / D=2$ to 2.5 , its convection velocity is about $0.45 U_{c}$. Downstream of $x / D>2.5$, the vortex is convecting at $U_{c}=0.62 U_{0}$. The value of convection velocity at $U_{c}=0.30 U_{0}$ in the very near wake may first appear to be too low. For vortices shed from a circular cylinder, it is well accepted and recently reported again in Zhou and Antonia ${ }^{12}$ that the convection velocity is of the range $0.8-0.9$ of the free-stream velocity. However, this value applied in the fully established wake beyond ten, or at least five diameters from the body. In the work of Cantwell and Coles, ${ }^{2}$ the convection speed reaches this value only downstream of four diameters, upstream of which the convection velocity drops rapidly. In the work of Leder ${ }^{3}$ on the near wake of flow past a vertical flat plate $(x / D<5)$, the convection velocity was not reported but a value of $U_{c}=0.27 U_{0}$ can be calculated from their data. Consequently, in this near wake of the inclined plate, a convection velocity of $U_{c}=0.30$ $U_{0}$ is found acceptable to be used to move an observer to visualize the early vortex dynamics. Actually, this convection velocity is not very incompatible with the mean flow velocity at the wake central axis which rises from near zero from $x / D=1.5$ to $0.35 U_{0}$ at $x / D=2.0$, and to $0.6 U_{0}$ at $x / D=3.0$.

Using $U_{c}=0.30 U_{0}$, the phase-locked flow vector patterns in Fig. 5 are transformed to those as seen by an observer moving with the vortices. These vector maps shown in Fig. 8 reveal more clearly the locations of the vortices and their associated fluid circulations. The development of vortices is more conveniently described from Figs. 5, 6, and 8 starting from phase 3. Here, a region of coherent fluid circulation can be observed behind the trailing side of the plate. This "vortex" of the counterclockwise sense is centered at $x / D=1.0$. At a farther distance, a shed vortex of the same sense and its associated fluid circulation can be found around $x / D=2.4$. Both vortices are connected with concentration of positive vorticity. At phase 4, the whole of the first vortex enters into the measurement region with its center shifted to $x / D=1.25$. It can be seen from Fig. 8 that the vortex induces strongly coherent counterclockwise fluid circulation around it. The associated intense concentration of positive vorticity is also clearly observed in Fig. 6. For the downstream vortex, its region of positive vorticity contours is observed in Fig. 6 to have moved to $x / D=2.5$. Figure 8 shows that it no longer induces fluid circulation around it. Instead, it induces fluid transport in the transverse direction at its leading and trailing fronts. This is the characteristic of a shed vortex after the supply of vorticity from the separation shear layer on the solid body has been cut off for some time. One may note that the convection speed of the vortex at $x / D$ around 2.5 has been found in Fig. 7 to be significantly higher than the observer speed used in Fig. 8. However, a number of published works have suggested that the dynamics of flow structures seen by a moving observer are largely insensitive to the exact value of the chosen observer speed. ${ }^{1}$ In the present study, some higher values of $U_{c}$ have been used to observe the convecting vortex patterns. The results, not shown here, confirm that after the vortex is fully shed, the vortex-induced fluid motions change from circulations around the vortex center to lateral transports at the vortex fronts, though observed to occur slightly farther downstream.

Development of the upstream counterclockwise vortex beyond $x / D>1.25$ can be observed from phases 5 to 8 in Figs. 5, 6, and 8. At phase 8, Fig. 6 shows that the vorticity hill is detached from the vorticity concentration located at $x / D<1$. If the latter vorticity concentration is due to the recirculation flow attached to the trailing edge of the plate, Fig. 6 shows that the shed vortex is now completely detached from the plate. The subsequent convection of the shed vortex can be observed at phases 1 to 2 and the vortex finally converges to the second counterclockwise vortex at phase 3 .

Looking at the value of peak vorticity associated with the counterclockwise vortex, it is evident from Fig. 6 that the vortex possesses very high vorticity values at phases 2 and 3 when it has not completely entered into the measurement region. It is probable that this early stage of the vortex is in the form of recirculating fluids still attached to the trailing edge of the plate. With the supply of vorticity fed from the separation shear layer at the plate trailing edge, the "standing" vortex continues to grow. At phase 3, the vortex has grown to having its center extended to an axial distance of $x / D=1.0$, which is $0.43 D$ downstream of the plate trailing edge. Its vorticity reaches a peak value of $\Omega D / U_{0}>8.0$. It is believed that the standing vortex is then detached from the plate and becomes a shed vortex. It is then cut off from the separation shear layer which feeds vorticity to it. This is suggested by the observation in Fig. 6 that the peak vorticity of the vortex remains at a fairly constant value of $\Omega D / U_{0} \simeq 3.3$ up to $x / D=2$. Farther downstream, diffusion 
Phase 1

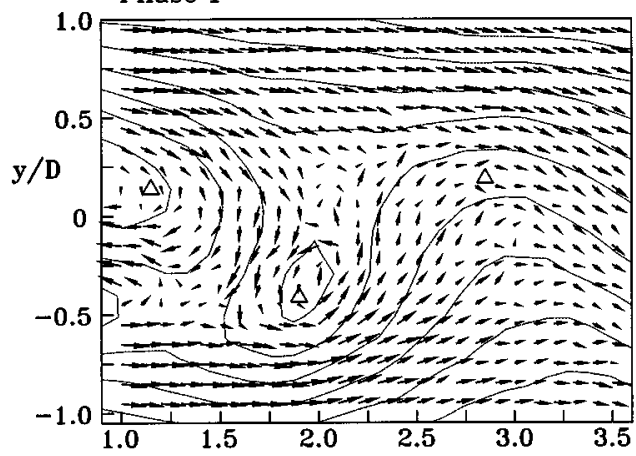

Phase 2

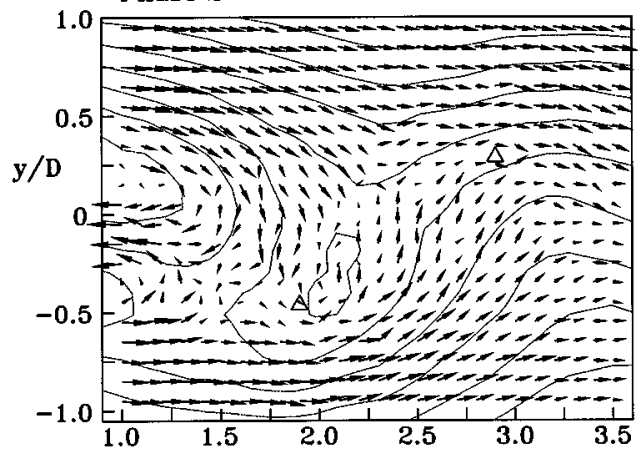

Phase 3
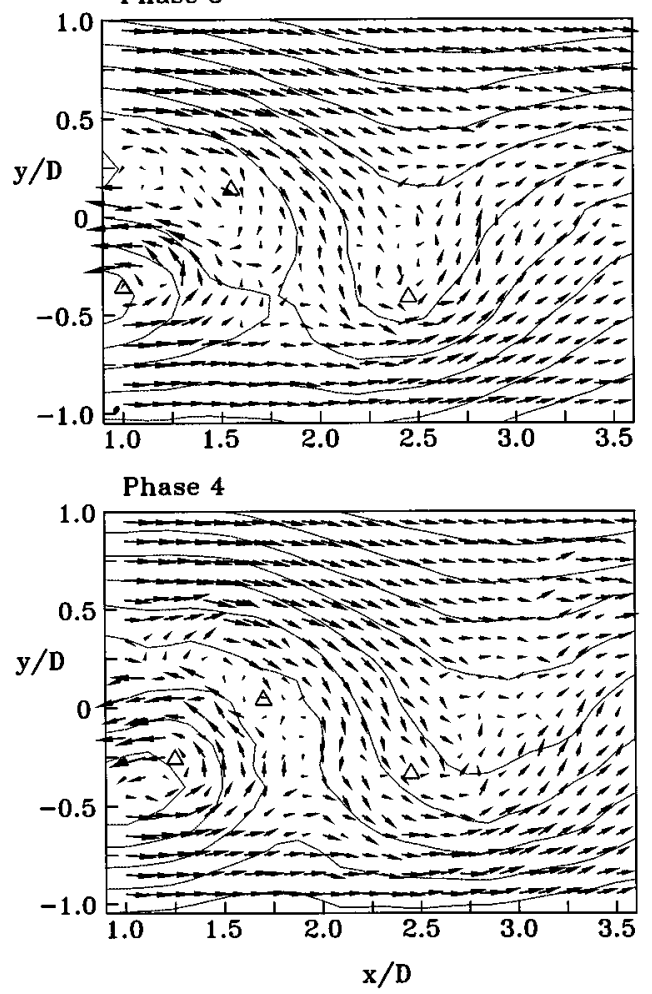

Phase 5

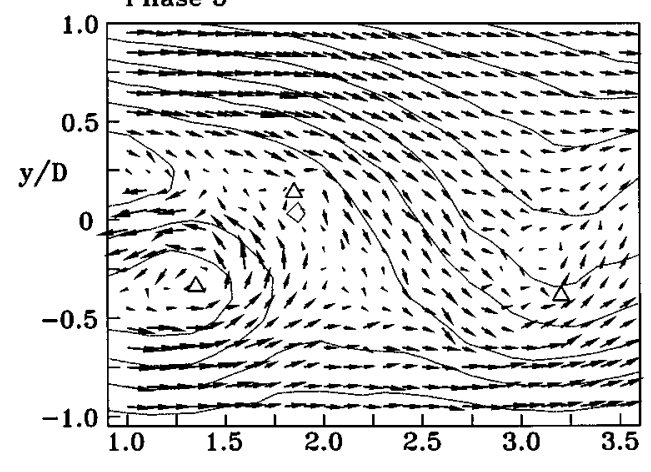

Phase 6

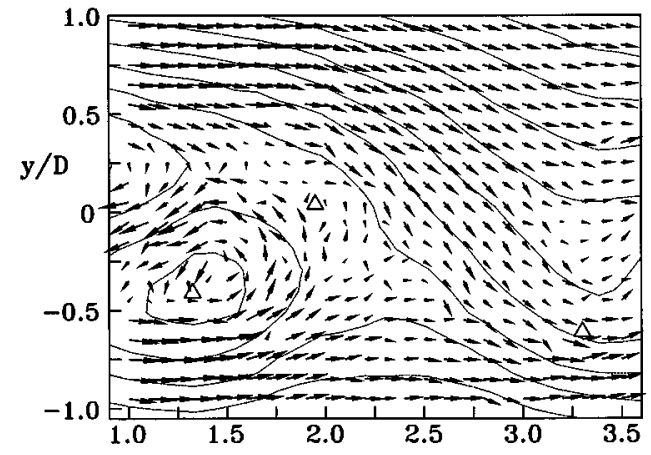

Phase 7

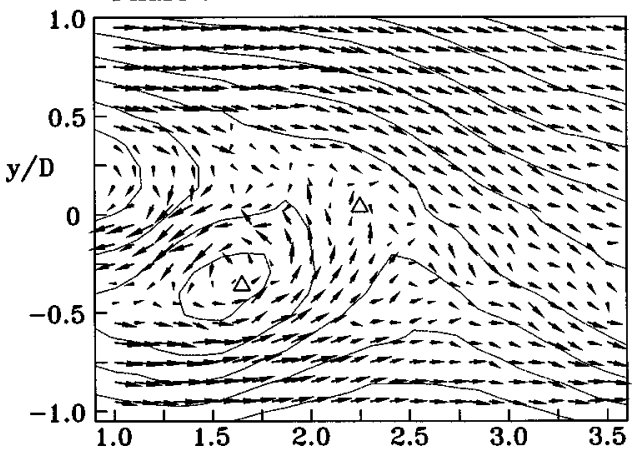

Phase 8

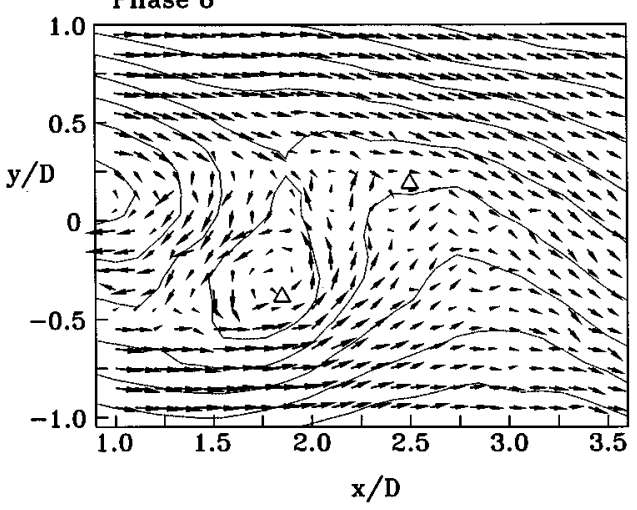

FIG. 8. Coherent flow patterns, as seen by an observer moving with the vortex at $U_{c}=0.3 U_{0}$. $\Delta$, location of peak positive or negative coherent vorticity.

of vorticity and coherent fluid circulation takes place and the peak vorticity drops significantly when the vortex is convected beyond $x / D>2$. Perry and Steiner ${ }^{7}$ investigated the vortex formation processes behind a flat plate at $\alpha=90^{\circ}$ and $45^{\circ}$. A noble apparatus was designed to allow velocity measurements with a flying hot wire at locations as far upstream as almost on the plate surface. The phase-averaged velocity vector plots presented for the inclined plate also showed that fluid circulation of a vortex reached its maximum when the vortex is just about being shed.

In flow past a circular cylinder or a vertical plate, vortices of equal strengths are shed alternatively from the two 
sides of the body. The shed vortices occupy a vortex street pattern of staggered arrangement. It seems that this arrangement of vortices is able to collaborate the transporting power of a pair of vortices of the opposite senses, one at its leading front and the other at its trailing front. As a result, the vortex street pattern sustains coherent large-extent transport of fluid across the whole wake in both lateral directions. With this lateral fluid transport, the vortices in the street seems to survive a farther distance. In Cantwell and Coles ${ }^{2}$ and Leder, ${ }^{3}$ vortices of good identity could be educed from phase averaging of the velocity field at axial distances beyond three or even more body dimensions. A relevant observation of the staggered vortex pattern was reported for the wake and wake-induced vortices in an annular jet by Lam et al. ${ }^{13} \mathrm{In}$ the present flow past an inclined plate, it is not evident from the educed flow patterns in Figs. 5, 6, and 8 that wellestablished clockwise vortices are shed from the leading edge of the plate. It cannot, of course, be ruled out the possible reason that the leading edge of the plate is much farther upstream of the beginning of the measurement region at $x / D=1$ so that the early development of counterclockwise vortices, if there are any, is missed. Anyway, at phase 7, the vector maps in Fig. 8 show observe some fluid circulation of the clockwise sense at a region following at the trailing edge of the main counterclockwise vortex. This "clockwise vortex" is centered at $x / D=1.0$ and $y / D=-0.25$. Figure 6 shows that it is connected with a concentration of negative vorticity and that this region of vorticity valley is convecting behind the main clockwise vortex. Not only that the peak value of negative vorticity is lower than the peak value of positive vorticity connected with the counterclockwise vortices, but also the region of concentration is more diffused. It is believed that this lack of a vortex street of staggered vortices of opposite vorticity and equal strength plays a key role in the short life span of the vortices in the present wake of an inclined plate at this small angle of attack.

The phase-averaged velocity vector fields of Perry and Steiner ${ }^{7}$ for a flat plate inclined at $45^{\circ}$ also showed that the vortices shed from the trailing edge induce stronger fluid circulation than those from the leading edge. Similar observations of a train of weaker vortices behind the leading edge could be found in the water-tunnel visualization pictures of McAlister and Carr $^{8}$ for an aerofoil at $\alpha$ pitching between $10^{\circ}$ and $20^{\circ}$. It appears that the asymmetry of the vortex street becomes more pronounced for an angle of attack at or below $30^{\circ}$.

The coherent Reynolds stresses connected with the vortical structures are shown in Fig. 9. These large-scale fluid transports are consequential to the dynamics of the coherent vortical structures, the centers of which are marked in Fig. 9 by the summit locations of vorticity hills and valleys in Fig. 6. As is common in wake flows, the coherent lateral velocity fluctuations $v_{c}=\langle V\rangle-\bar{V}$ are much larger than the coherent streamwise turbulence intensity $u_{c}=\langle U\rangle-\bar{U}$. This anisotropy of turbulence intensities has been reported in the wake of a normal flat plate by Bradbury ${ }^{14}$ in which it was argued to be a feature of shedding of a vortex street. Figure 9(a) shows that the counterclockwise vortex transports fluids laterally upwards at its leading front and entrains fluids down- wards at its trailing front. The former transport activity at the leading front is clearly stronger. It is also obvious that the $v_{c}$ contours are organized in pairs of a negative $v_{c}$ valley following a positive $v_{c}$ hill. This is due to the dominance of the counterclockwise vortices over the "clockwise vortices." Figure 9(b) shows the streamwise velocity fluctuations resulting from the lateral fluid movements. As the counterclockwise vortex transports fluid upwards at its leading front, fast moving fluids in the free stream are entrained at the lower region of the wake. This explains the small positive $u_{c}$ hills to the lower right side of the vortex center. In the upper part of the vortex's leading front, low velocity fluids are ejected from the wake into the free stream, leading to the small negative $u_{c}$ valley to the upper right side of the vortex. The same dynamics occur at the trailing front of the vortex. Figure 9(c) shows the coherent Reynolds stress contours of $u_{c} v_{c}$. The contours exhibit a four-lobe appearance which is a consequence of the two-lobe and four-lobe characteristics, respective of the $v_{c}$ and $u_{c}$ contours. The dominance of the counterclockwise vortex in the coherent fluid dynamics is evident from these Reynolds stress contours. Two hills of concentrated positive $u_{c} v_{c}$ cluster around the lower side of the vortex while there are two valleys of negative coherent Reynolds stress at its upper side.

Contour plots of the phase-averaged Reynolds stresses have been reported for a normal flat plate in Perry and Steiner ${ }^{7}$ and Leder. ${ }^{3}$ In both studies, the phase-averaged total shear stress was presented as $\left\langle u^{\prime} v^{\prime}\right\rangle$ which is $\left\langle\left(u_{c}+u_{r}\right)\left(v_{c}+v_{r}\right)\right\rangle$. The contour plots of the former study appear to be of higher precision and show a clear symmetrical four-lobed distribution. In the present study, coincident measurement of $u^{\prime}$ and $v^{\prime}$ was not possible due to the sharing of single-channel capability of the LDA processor. As a result, only the coherent shear stress $u_{c} v_{c}$ is available and this differs from $\left\langle u^{\prime} v^{\prime}\right\rangle$ by the omission of the incoherent Reynolds stress $\left\langle u_{r} v_{r}\right\rangle$. The significance of this incoherent shear stress was discussed in Hussain ${ }^{1}$ and was argued to play an important role in the dynamics of coherent structures undergoing strong interactions, such as pairing. In weaker interactions like vortex convection in the present wake, the significance of the incoherent term is not known. The maximum magnitude of coherent stress in Fig. 9(c) is about $u_{c} v_{c} / U_{0}^{2}>0.05$, while the maximum total stress for the normal flat plate was reported in Perry and Steiner ${ }^{7}$ to be about $\left\langle u^{\prime} v^{\prime}\right\rangle / U_{0}^{2}>0.12$. The difference in plate inclination angle in the two studies renders a direct comparison of the stress values difficult. The significance of the incoherent shear stress in flat plate wakes remains an unresolved problem and may call for future investigations.

It is clear in Fig. 9 that the important activities of fluid transport and Reynolds stress production occur mainly in the lower part of the wake where the counterclockwise vortices convect. Figures 5, 6, and 8 show that the counterclockwise vortex convects in this region with its center lying between $y / D=-0.3$ and -0.4 . The region of fluid circulation of the clockwise sense is found to convect with its center lying between $y / D=0$ and 0.2 . This shows that the asymmetric vortex street is lying more towards the lower side of the wake, that is the side behind the plate trailing edge. The 


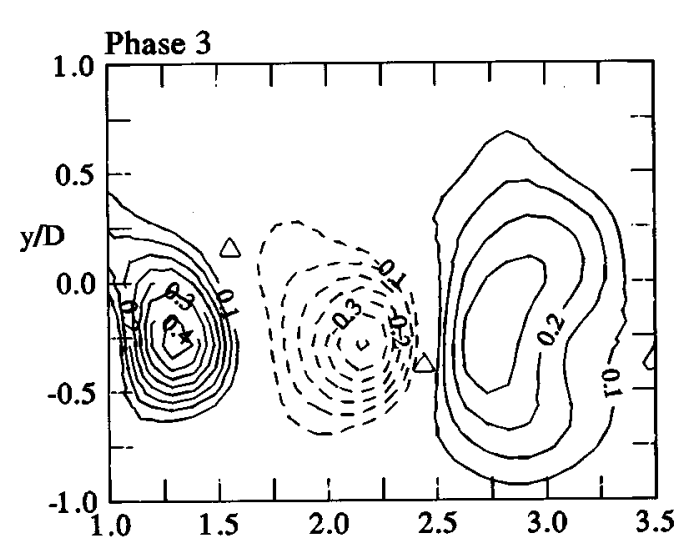

(a) $v_{c}$
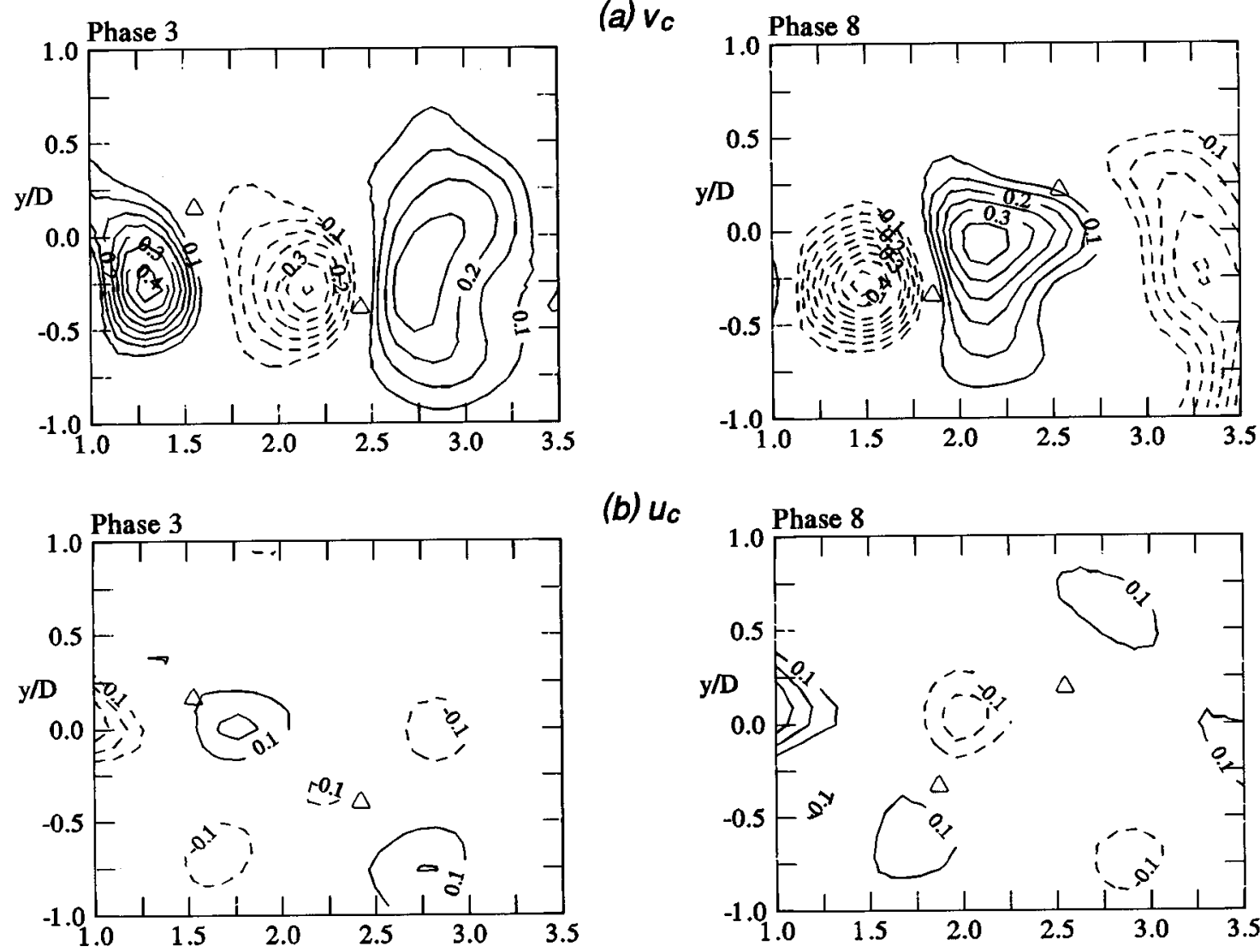

(b) $u_{c}$
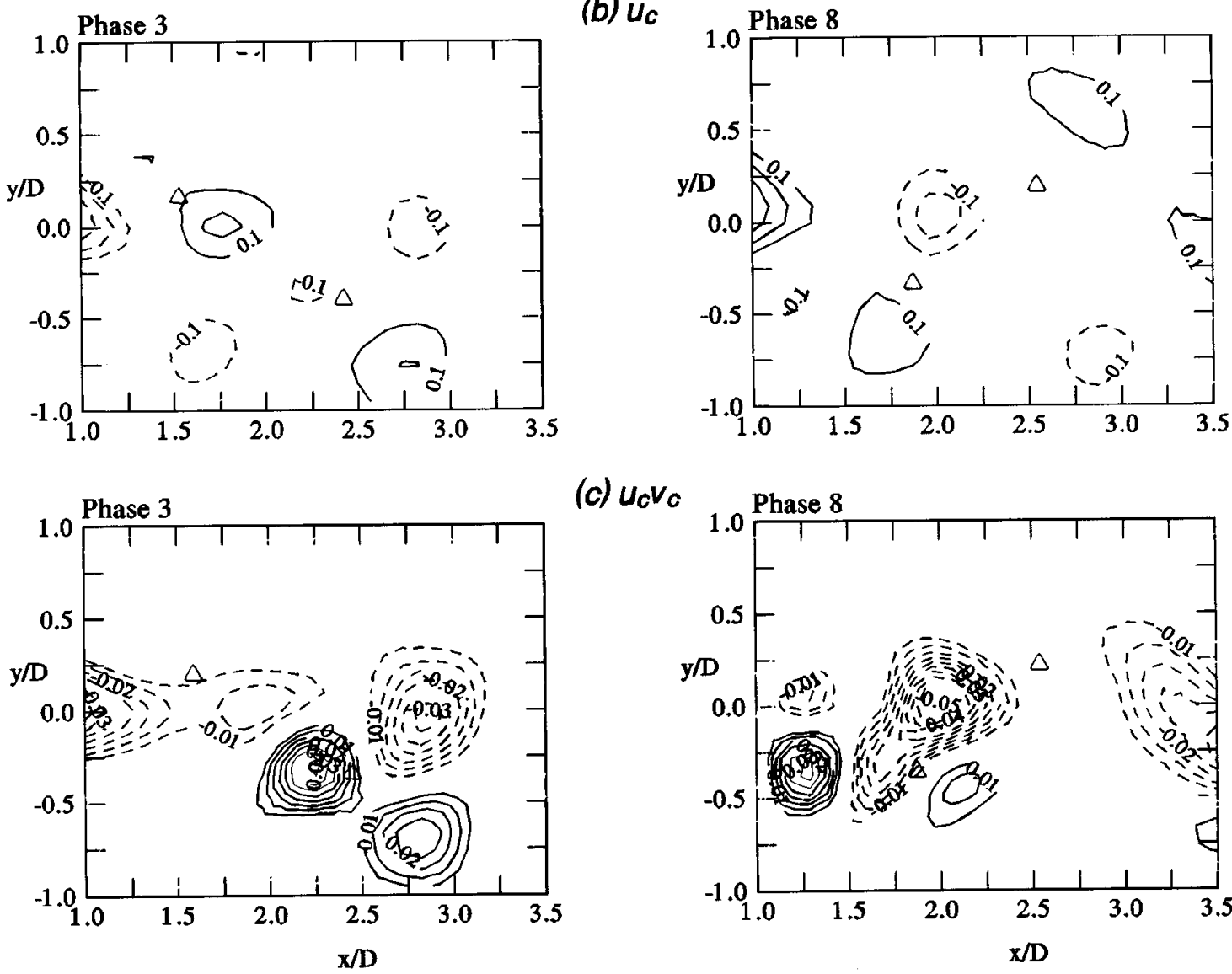

(c) $u_{c} v_{c}$

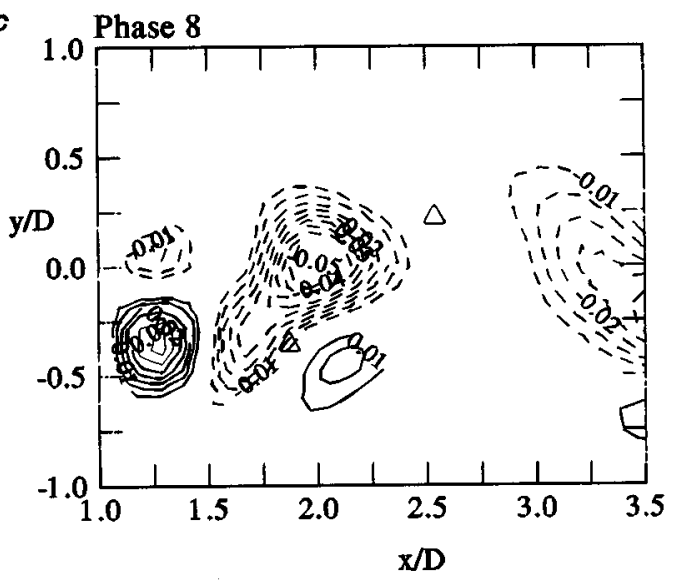

FIG. 9. Contours of coherent Reynolds stresses. (a) coherent lateral velocity component, $v_{c}=\langle V\rangle-\bar{V}$. Levels in $v_{c} / U_{0}$; (b) coherent streamwise velocity component, $u_{c}=\langle U\rangle-\bar{U}$. Levels in $u_{c} / U_{c}$; (c) coherent Reynolds stress, $u_{c} v_{c}$. Levels in $u_{c} v_{c} / U_{0}^{2} . \Delta$, location of peak positive or negative coherent vorticity.

same feature was observed from flow visualizations made behind an aerofoil under dynamic stall. ${ }^{8}$ This location of the vortex-street wake further supports the dominance of the counterclockwise vortices in the wake. It may be worthwhile here to return to the phase-averaged streamline patterns in Figs. 5 and 8 and note how they reveal an obvious dominance of the counterclockwise vortices from the trailing edge of the plate.

\section{CONCLUSIONS}

Velocity signals in the wake of an inclined flat plate at $\alpha=30^{\circ}$ are measured with an LDA. The coherent flow struc- tures in the wake and their dynamics are educed with the phase-locked averaging technique. It is found that the wake is dominated by a train of counterclockwise vortices shed from the trailing edge of the plate. The vortices are associated with concentration of coherent vorticity and large-scale production of Reynolds stresses. There is evidence that coherent vorticity is accumulated in the vortices while they are still attached to the plate and being fed vorticity by the separation shear layer. After the vortices are shed, diffusion of vorticity takes place while they are convected downstream. The asymmetric configuration and the small angle of attack of the inclined plate does not seem to support the formation 
of a well-balanced vortex street of opposite sign vortices of the same strength. Some coherent fluid circulation of the clockwise sense is detected on the side of the wake behind the plate leading edge, but the eduction results cannot show the definite existence of coherent clockwise vortices shed from the leading edge. The educed coherent vorticity and Reynolds stress production associated with these clockwise fluid motions are of lesser significance than those of the counterclockwise vortices. When compared with the vortical structures behind a circular cylinder or a normal flat plate, the large-scale vortices in the presence wake are observed to decay more rapidly. This may be argued to be related to the lack of a well-balanced vortex street pattern.

\section{ACKNOWLEDGMENTS}

An early version of this paper was presented at the First International Conference on Flow Interaction, The University of Hong Kong, Hong Kong, 5-9 September 1994. The author wishes to thank Mr. Y. Xan which carried out the LDA measurements. Mr. Xan is with the Mechanical Engineering Department, Research Institute of Petroleum Exploration and Development, Beijing, People's Republic of China. The investigation is supported partly by a research grant provided by the University of Hong Kong.

${ }^{1}$ A. K. M. F. Hussain, “Coherent structures—reality and myth,' Phys. Fluids 26, 2816 (1983).
${ }^{2}$ B. Cantwell and D. Coles, "An experimental study of entrainment and transport in the turbulent near wake of a circular cylinder,' J. Fluid. Mech. 136, 221 (1983).

${ }^{3}$ A. Leder, "Dynamics of fluid mixing in separated flows," Phys. Fluids A 3, 1741 (1991).

${ }^{4}$ A. Fage and F. C. Johansen, " On the flow of air behind an inclined flat plate of infinite span," Proc. R. Soc. London Ser. A 116, 170 (1927).

${ }^{5} \mathrm{C}$. W. Knisely, "Strouhal numbers of rectangular cylinders at incidence: a review and new data,' J. Fluids Struct. 4, 371 (1990).

${ }^{6} \mathrm{~T}$. Sarpkaya, "An inviscid model of two-dimensional vortex shedding for transient and asymptotically steady separated flow over an inclined plate," J. Fluid Mech. 68, 109 (1975).

${ }^{7}$ A. E. Perry and T. R. Steiner, "Large-scale vortex structures in turbulent wakes behind bluff bodies. Part 1. Vortex formation processes," J. Fluid Mech. 174, 233 (1987).

${ }^{8} \mathrm{~K}$. W. McAlister and L. W. Carr, "Water-tunnel visualizations of dynamic stall," in ASME Symposium on Nonsteady Fluid Dynamics, 1979 Winter Meeting, New York, 2-7 December 1979, edited by D. E. Crow and J. A. Miller (ASME, New York, 1979), p. 103.

${ }^{9}$ E. Naudascher and D. Rockwell, Flow-Induced Vibrations: An Engineering Guide (A.A. Balkema, Rotterdam, 1994), p. 195.

${ }^{10}$ J. R. Calvert, "Experiments on the flow past an inclined disk," J. Fluid Mech. 29, 697 (1967).

${ }^{11} \mathrm{~J}$. Li, J. Sun, and B. Roux, "Numerical study of an oscillating cylinder in uniform flow and in the wake of an upstream cylinder,' J. Fluid Mech. 237, 457 (1992).

${ }^{12} \mathrm{Y}$. Zhou and R. A. Antonia, "Convection velocity measurements in a cylinder wake,' Exp. Fluids 13, 63 (1992).

${ }^{13}$ K. M. Lam, N. W. M. Ko, and K. K. Lau, "Wake and wake-induced shear-layer excitation in an annular jet," Phys. Fluids 29, 3121 (1986).

${ }^{14}$ J. S. Bradbury, "Measurement with a pulsed-wire and a hot-wire anemometer in the highly turbulent wake of a normal flat plate,' J. Fluid Mech. 77, 473 (1976) 